\title{
QD4G: QoE para Vídeo em Redes D2D/4G com Aprendizado de Máquina
}

\author{
Marcos Carvalho $^{1}$, Erik de Britto e Silva ${ }^{1}$, Vinicius F. Silva ${ }^{1}$, \\ Daniel F. Macedo ${ }^{1}$
}

\author{
${ }^{1}$ Departamento de Ciência da Computação \\ Universidade Federal de Minas Gerais (UFMG) - Belo Horizonte, MG - Brasil \\ \{marcoscarvalho, erik, viniciusfs, damacedo\}@dcc.ufmg.br
}

\begin{abstract}
Device-to-Device (D2D) communication is one of the mechanisms to offload $4 G$ networks. Hybrid D2D/4G networks have the challenge to multiplex efficiently the communication interfaces, which impacts directly the user's Quality of Experience ( $Q o E)$. We present $Q D 4 G$, a platform that employs Machine Learning to predict when clients using the DASH (Dynamic Adaptive Streaming over HTTP) standard must use the $4 G$ network or the D2D network. The goal is to improve the QoE providing better video streaming resolution. Experiments with real devices show that prediction achieves an accuracy of up to $87 \%$, increasing the mean video resolution among users over D2D by up to $150 \%$.
\end{abstract}

Resumo. Comunicação Dispositivo-a-Dispositivo (D2D) é um dos mecanismos para alivio das redes $4 G$, via offload de dados. Redes híbridas D2D/4G possuem o desafio de multiplexar com eficiência as interfaces de comunicação, o que impacta a Qualidade de Experiência (QoE) dos usuários. Apresentamos o QD4G, uma plataforma que usa Aprendizado de Máquina para predizer quando clientes utilizando o padrão DASH (Dynamic Adaptative Streaming over HTTP) devem utilizar a rede $4 G$ ou a rede D2D. O objetivo é melhorar o QoE fornecendo vídeo em melhor resolução. Experimentos com dispositivos reais mostram que a predição atinge até $87 \%$ de acerto, aumentando a resolução média do vídeo via $\mathrm{D} 2 \mathrm{D}$ em até $150 \%$.

\section{Introdução}

A alta demanda por vídeo, juntamente com outros serviços em execução concorrente nas redes 4G, limita a vazão disponível para cada fluxo. Padrões de transmissão adaptativa de vídeo, como o DASH (Dynamic Adaptative Streaming over HTTP) [Stockhammer 2011], reduzem a qualidade do vídeo em situações de baixa vazão. Um vídeo de menor resolução, embora permita que a mídia ainda seja reproduzida, pode resultar em usuários insatisfeitos com a menor resolução.

Existe um crescimento constante do número de assinantes móveis, bem como de novos aplicativos de uso intensivo de dados, como por exemplo jogos online e compartilhamento de vídeo na Internet [Thrimurthulu and Sarma 2017]. Esse crescimento coloca em risco a atual capacidade das redes celulares, em especial as redes Long Term Evolution (LTE), conhecidas como redes $4 \mathrm{G}$, que operam cada vez mais perto de seus limites.

Diante disso, a indústria e a comunidade científica têm proposto novos paradigmas para aliviar a congestão nas redes $4 \mathrm{G}$. Um desses paradigmas é o descarregamento de dados das redes $4 \mathrm{G}$, que vão do uso de estações celulares pequenas, de baixa 
potência, conhecidas como femtocélulas [Haldar et al. 2013], às redes Wi-Fi públicas [Mota et al. 2013] e, recentemente, a comunicação Dispositivo-a-Dispositivo (Device-toDevice - D2D) [Doppler et al. 2009].

A comunicação D2D move o tráfego de dados das redes celulares para outras interfaces de comunicação, processo conhecido como mobile data offloading [Zhou et al. 2018]. Redes D2D podem ser inband quando utilizam o espectro licenciado para conectar os dispositivos, ou outband, quando utilizam as outras faixas de uso não licenciado do espectro (por exemplo, empregando tecnologias como o Wi-Fi, Zigbee e Bluetooth ${ }^{2}$ ).

Em D2D, diferentes usuários requisitando o mesmo conteúdo poderão compartilhálo entre si, dispensando a necessidade de várias requisições de um mesmo conteúdo na rede infraestruturada. Um exemplo seria torcedores em um estádio vendo o replay de uma jogada nos seus celulares. Na rede $4 \mathrm{G}$ tradicional, o consumo de banda no núcleo da rede da operadora seria proporcional à quantidade de usuários simultâneos assistindo o vídeo. Ao empregarmos D2D, este mesmo vídeo poderia ser transmitido somente uma vez no núcleo da rede, e em seguida seria compartilhado entre os usuários empregando a interface sem fio para a comunicação D2D.

Além de uma maior qualidade de experiência (Quality of Experience - QoE), o uso de uma rede D2D gera outros ganhos, tais como um menor consumo de energia [Xu et al. 2018], maiores taxas de transmissão [Chai et al. 2016] e diminuição do consumo de banda na rede 4G, contribuindo para a desoneração da rede [Pyattaev et al. 2013].

Um dos desafios da comunicação híbrida 4G/D2D é a identificação de quando uma rede D2D deve ser criada, fator que irá definir todo o desempenho dos usuários a partir de um determinado momento. Ainda que a comunicação D2D tenha um desempenho médio superior à comunicação 4G, o emprego dessa forma de comunicação em condições inadequadas (Ex.: Usuários distantes um do outro, faixa de frequência sujeita a interferências de outras redes, presença de obstáculos) pode trazer prejuízos ao QoE.

Este trabalho apresenta uma plataforma denominada $Q D 4 G$, que identifica quando criar novos grupos D2D para suportar transmissões de vídeo usando o padrão DASH. A decisão utiliza um preditor baseado em Aprendizado de Máquina (AM) para maximizar o QoE do usuário, ao maximizar a resolução do vídeo sendo transmitido. Assim, um novo grupo D2D é criado quando o preditor identifica que os usuários receberiam um vídeo de maior qualidade ao usar a rede D2D ao invés da rede 4G. A solução proposta utiliza Wi-Fi Direct para construir redes D2D outband. As principais contribuições deste trabalho são:

- Desenvolvimento de um preditor que avalia a resolução esperada do vídeo, com base no tráfego de cada usuário conectado na rede $4 \mathrm{G}$;

- Uma avaliação da eficácia do preditor em dispositivos reais em um testbed empregando 4G e Wi-Fi Direct.

Os resultados experimentais mostraram que a predição permite atingir $87 \%$ de acerto, com aumento na resolução média do vídeo entre os usuários via D2D de até $150 \%$. Até onde sabemos, este é o primeiro trabalho que propõe o emprego de comunicação D2D com base em previsões da resolução do vídeo em cada usuário, ao mesmo tempo quantificando em dispositivos reais o desempenho do preditor proposto.

\footnotetext{
${ }^{1} \mathrm{https}: / /$ www.zigbee.org - Último acesso em 28/02/2019

${ }^{2}$ https://www.bluetooth.org - Último acesso em 28/02/2019
} 
O restante deste trabalho está organizado da seguinte forma: A seção 2 apresenta o referencial teórico. A seção 3 apresenta os trabalhos relacionados. A seção 4 apresenta a plataforma QD4G. A seção 5 descreve a metodologia empregada nos experimentos e os resultados obtidos. A seção 6 conclui este trabalho e apresenta os trabalhos futuros.

\section{Referencial Teórico}

Esta seção apresenta os principais conceitos utilizados neste trabalho. Inicialmente são apresentadas as redes celulares 4G LTE, seguida de uma visão geral da comunicação D2D integrada às redes 4G. A seguir é apresentada a tecnologia Wi-Fi Direct. Por fim, é apresentado o padrão DASH, utilizado neste trabalho para a transmissão de vídeo.

\subsection{G LTE}

A arquitetura das redes 4G, também conhecidas como LTE (Long Term Evolution), proposta pelo 3GPP (3rd Generation Partnership Project) [Sesia et al. 2009], é dividida em duas partes principais. A primeira é o UE (User Equipment), que na prática são os dispositivos dos usuários finais (Ex.: Smartphones, tablets, etc.). Já a segunda parte, chamada de EPC (Evolved Packet Core), são as estações rádio base, também conhecidas como eNodeB (Evolved Node Base Station), que utilizam IP e redes cabeadas para conectar o UE à Internet [Bradai et al. 2015]. A Figura 1 apresenta uma ilustração da arquitetura LTE.

O EPC é subdividido em diferentes módulos e serviços, conectados em sequência de forma a processar adequadamente os pacotes enviados e recebidos, e coletar as informações necessárias de cada UE. Os principais módulos são o S-GW (Serving Gateway), o P-GW (Package Data Network Gateway), o MME (Mobility Management Entity), o PCRF (Policy and Charging Rules Function) e o HSS (Home Subscriber Server).

Os eNodeBs se conectam ao MME através de uma interface cabeada por meio do protocolo SCTP, sendo que essa interface é utilizada para trocar dados de autenticação dos UEs. Os eNodeBs também conectam-se diretamente ao $S-G W$ por meio de um túnel GTP, sendo que o S-GW funciona como um roteador de tráfego na questão da mobilidade dos UEs entre eNodeBs. O S-GW também é responsável pelo encaminhamento de dados de aplicação ao P-GW, que por sua vez os encaminha à Internet. Já o PCRF define políticas e decisões de gerenciamento, tal como a alocação de IP aos UEs. Por fim, o HSS fornece diferentes informações relacionadas aos UEs, as quais podem ou não ser utilizadas pelo MME [Mi et al. 2014].

\subsection{Comunicação Dispositivo-a-Dispositivo}

O padrão 3GPP Device-to-Device Proximity Services (D2D ProSe) define como comunicação D2D a comunicação ad hoc entre dispositivos próximos, com mínima ou nenhuma intervenção da estação-base [Asadi et al. 2014]. O D2D ProSe utiliza o Long Term Evolution-Advanced (LTE-A) como tecnologia de rádio para comunicação ad hoc (comunicação inband). No entanto, o D2D Prose também pode explorar o espectro não licenciado para a comunicação ad hoc entre dispositivos (comunicação outband). Neste caso, são empregadas tecnologias como Bluetooth, Wi-Fi em modo ad-hoc ou Wi-Fi Direct para construir a rede D2D outband. Grupos D2D podem ser criados através de comandos das operadoras de celular. 


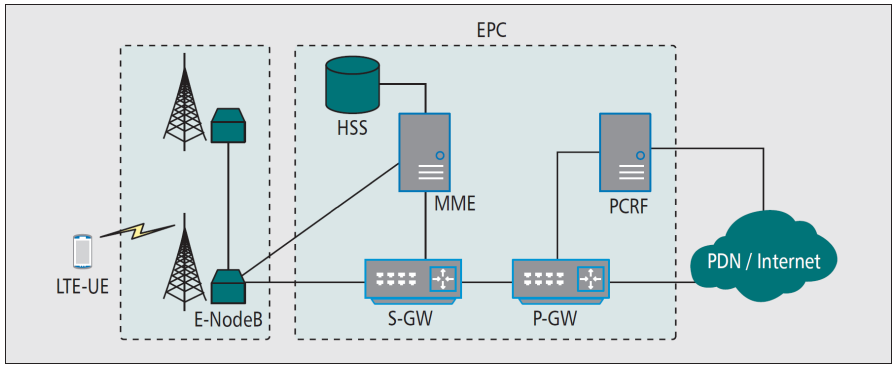

Figura 1. Arquitetura da Rede 4G LTE (adaptado de [Bradai et al. 2015]).

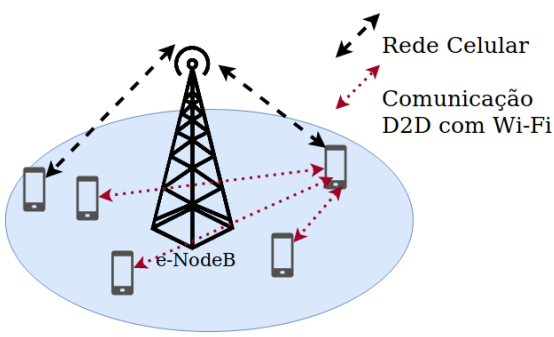

Figura 2. Comunicação D2D outband usando Wi-Fi Direct.

A comunicação D2D ocorre diretamente entre os UEs, como é mostrado na Figura 2, ao contrário da comunicação tradicional, que ocorre entre os UEs e o eNodeB. Em situações onde $n$ UEs estão solicitando o mesmo conteúdo, o D2D pode reduzir o tráfego na interface celular para até $1 / n$ do tráfego original. Para tanto, é comum associarmos o redirecionamento dos dados a grupos D2D com o caching de dados nos UEs.

A comunicação D2D foi desenvolvida tendo em vista um conjunto de aplicações, a saber: Comunicação entre dispositivos em situações de emergência ou desastre; Aumentar a área de cobertura da rede $4 \mathrm{G}$, pois usuários distantes podem repassar seus dados via dispositivos de usuários conectados à antena; Descarregamento de dados da rede celular para a rede D2D, aumentando a eficiência espectral ao empregarmos uma comunicação entre dispositivos mais próximos; Serviços localizados, como por exemplo IoT, indicações de estacionamento e marketing geo-localizado.

\subsection{Wi-Fi Direct}

Em Outubro de 2010, Wi-Fi Alliance ${ }^{3}$ lançou o Wi-Fi Direct ${ }^{4}$ para dispositivos que suportam comunicação ad-hoc via Wi-Fi. O Wi-Fi Direct simplifica a conexão de dispositivos sem fio, permitindo que se comuniquem diretamente um com o outro, sem um ponto de acesso, com a segurança garantida através do padrão WPA2.

A conexão entre dois ou mais dispositivos forma um "grupo D2D”, sendo que cada grupo D2D possui um ou mais clientes e um dispositivo especial denominado Group $O w$ ner (GO), responsável pela autenticação de novos clientes, pelo encerramento do grupo em si, e também pelo acesso dos clientes à Internet. Dispositivos sem fio que não possuam suporte à comunicação via D2D Wi-Fi Direct, denominados "Dispositivos Legados" (Legacy Devices) também podem se conectar através do GO, o qual também emula um ponto de acesso tradicional [Camps-Mur et al. 2013].

\subsection{DASH - Protocolo Padrão para Streaming de Vídeo}

O DASH (Dynamic Adptive Streaming over HTTP) é um protocolo padrão para streaming de vídeo iniciado pelo 3GPP, que visa resolver as limitações encontradas nas soluções de vídeo tradicionais, por exemplo as baseadas em RTSP (Real-Time Streaming Protocol), que necessitam de um monitoramento constante do status do usuário durante o download do vídeo [Stockhammer 2011]. O DASH também visa resolver limitações de

\footnotetext{
${ }^{3}$ https://www.wi-fi.org - Último acesso em 28/02/2019

${ }^{4}$ https://www.wi-fi.org/discover-wi-fi/wi-fi-direct/ - Último acesso em 28/02/2019
} 
soluções baseadas em download de vídeo progressivo através de HTTP, que não consideram a demanda específica do usuário ao longo do tempo (ex.: acesso aleatório a determinados trechos do vídeo), que desperdiça banda com os trechos de vídeo não consumidos.

A principal característica do DASH é o ajuste da resolução do vídeo em tempo real pelo usuário, de acordo com métricas de rede tais como a vazão, por exemplo. Nas soluções tradicionais, a falta desse mecanismo faz com que o QoE do usuário seja ameaçado em cenários com grande demanda, já que o vídeo é requisitado em uma resolução imutável e com isso poderá ser interrompido, devido à variação no atraso ou na banda. Outra característica do DASH é que o vídeo é carregado em partes (chunks) contendo alguns segundos, ao invés de fluxos contínuos de pacotes como é feito tradicionalmente.

O ajuste de resolução em tempo real no DASH é um tema ainda explorado na literatura $^{5}$. Kua et al. [Kua et al. 2017] citam as principais técnicas de ajuste de vazão no DASH, e as avaliam do ponto de vista do cliente, do servidor e da rede como um todo.

\section{Trabalhos Relacionados}

$\mathrm{Na}$ literatura, a avaliação de propostas para seleção de nós de retransmissão, criação de grupos D2D e aplicações são em sua maioria trabalhos de análises teóricas ou de simulações [Liu et al. 2015, Asadi et al. 2014], diferentes do presente trabalho, realizado em testbed físico. Alguns trabalhos visam a eficiência do espectro da rede celular ou avaliam alguns casos de uso, como comunicação multicast e aplicações peer-to-peer.

Ullah e Hong [Ullah and Hong 2016] escolhem como compartilhador via D2D o UE com maior SNR em relação ao eNodeB. Nessa proposta, um UE anuncia os arquivos de seu interesse e um UE vizinho realizará o compartilhamento, mesmo que esse ainda esteja realizando o download dos mesmos. A proposta dos autores difere do presente trabalho uma vez que o presente emprega um orquestrador baseado em AM, que comanda a troca de interface nos UEs com base na previsão de resolução do vídeo nos UEs. Além disso, o trabalho acima foi limitado somente em simulação.

Essaili et al. [Essaili et al. 2015] propõem a gerência de recursos de transmissão baseada no espaço dos buffers nos UEs. O QoE é avaliado por meio da vazão de transmissão, obtida por serviços próximos ao eNodeB. O trabalho otimiza os recursos da rede celular ao dirigir mais tráfego para os UEs com buffers vazios. Uma das principais limitações do trabalho é que melhorias na vazão são obtidas por traffic shapping. Como consequência, não efetua offloading da rede 4G, desvantagem perante o presente trabalho que propõe um modelo de predição para formar grupos D2D, que efetuam offloading.

Doppler et al. [Doppler et al. 2009] aumentam a vazão de downlink através de D2D underlay. Os autores propõem um controle do canal D2D, porém entre apenas dois dispositivos, ao invés de grupos de tamanho arbitrário. O tráfego via $4 \mathrm{G}$ de cada UE é monitorado para a possível formação de canal D2D entre dois UEs. O sistema implementado decide utilizar a comunicação D2D sempre que houver um ganho de vazão em tal comunicação frente à $4 \mathrm{G}$. A proposta ainda controla o nível da potência de transmissão entre os transmissores D2D, para diminuir a interferência dos mesmo sobre os UEs 4G. Tal trabalho limita o offloading a 50\%, desvantagem perante o presente trabalho que

\footnotetext{
${ }^{5}$ A busca pelo termo DASH QoE na base de dados do IEEE Xplore retornou mais de 100 trabalhos publicados de 2017 a 2018.
} 
permite a formação de grupos com mais de dois UEs.

Pyattaev et al. [Pyattaev et al. 2013] calcularam a viabilidade do offloading de dados móveis com Wi-Fi Direct. O gerenciamento da formação de grupo fica por conta da rede celular, que considera a localização dos dispositivos. Os autores mostram que os clientes D2D obtêm melhores taxas de transferência devido aos enlaces mais curtos. Como desvantagem, há o emprego de um simulador.

Os trabalhos mencionados acima são sumarizados na Tabela 1. Como vemos, a literatura carece de uma avaliação da transmissão de vídeos utilizando o DASH sob demanda em redes 4G/D2D em ambiente experimental, como é feito neste trabalho.

Tabela 1. Comparativo com Trabalhos Relacionados

\begin{tabular}{|c|c|c|c|c|c|c|}
\hline REFERÊNCIA & $\begin{array}{c}\text { AVALIA } \\
\text { QoE? }\end{array}$ & $\begin{array}{c}\text { CONSIDERA } \\
\text { VÍDEO? }\end{array}$ & $\begin{array}{c}\text { UTILIZA } \\
\text { D2D? }\end{array}$ & $\begin{array}{c}\text { AMBIENTE DE } \\
\text { AVALIAÇÃO }\end{array}$ & $\begin{array}{c}\text { GRUPOS OU } \\
\text { PARES D2D }\end{array}$ & $\begin{array}{c}\text { LOCAL DO } \\
\text { CONTROLE }\end{array}$ \\
\hline [Ullah and Hong 2016] & SIM & SIM & SIM & SIMULADOR & PAR & UEs \\
\hline [Essaili et al. 2015] & SIM & SIM & NÂO & SIMULADOR & NÂO & REDE \\
\hline [Doppler et al. 2009] & NÂO & MISTA & SIM & SIMULADOR & PAR & REDE \\
\hline [Pyattaev et al. 2013] & NÃO & NÃO & SIM & SIMULADOR & PAR & REDE \\
\hline QD4G & SIM & SIM & SIM & TESTBED & GRUPO & REDE \\
\hline
\end{tabular}

\section{A Plataforma QD4G}

O QD4G (QoE-based DASH video over 4G) é uma plataforma que define quando usar a rede 4G ou D2D para transmitir vídeos DASH, para maximizar o QoE dos clientes. A decisão é tomada através da predição de QoE: Se o QoE (neste caso, a resolução horizontal do vídeo) é aumentado utilizando D2D, então a rede $4 \mathrm{G}$ instrui os UEs para formarem grupos D2D.

A plataforma é composta por três partes: Um modelo de predição de QoE, um módulo de controle de conexão $\mathrm{D} 2 \mathrm{D}$, com o algoritmo de decisão que recebe as predições do modelo, e um software de reprodução de vídeo baseado em DASH, que suporta D2D. A Figura 3 mostra uma arquitetura que não emprega comunicação D2D (primeira parte) comparada com uma arquitetura que emprega D2D através da plataforma QD4G (segunda parte). Na primeira parte, os UEs realizam o download do vídeo em diferentes resoluções e o armazenam em seu respectivo cache. Já na segunda parte, com o modelo de predição ativo, é possível prever qual UE terá a maior resolução horizontal do vídeo, fato que é informado ao módulo de controle de conexão D2D. No exemplo apresentado na Figura 3, o GO escolhido é o UE 3, que transfere vídeo com maior QoE para os clientes.

Apesar de utilizar a resolução horizontal do vídeo como métrica de QoE, é importante salientar que o método não requer nenhuma informação dos UEs durante sua execução. Isto ocorre porque o dado da resolução obtida é utilizado somente no treinamento do preditor.

\subsection{Modelo de Predição}

A predição desenvolvida emprega $\mathrm{AM}$ para prever qual seria o QoE do vídeo quando transmitido em uma rede 4G. Consideramos como indicador de QoE a resolução horizontal do vídeo transmitido. Assim não são utilizados alguns métodos de QoE existentes que são impraticáveis para redes de produção, pois os usuários devem classificar o vídeo em uma escala de qualidade, o que é tedioso e pode fazer com que o usuário desista de assistir. Ao usar a resolução como métrica de QoE, apesar de não considerar todas as 


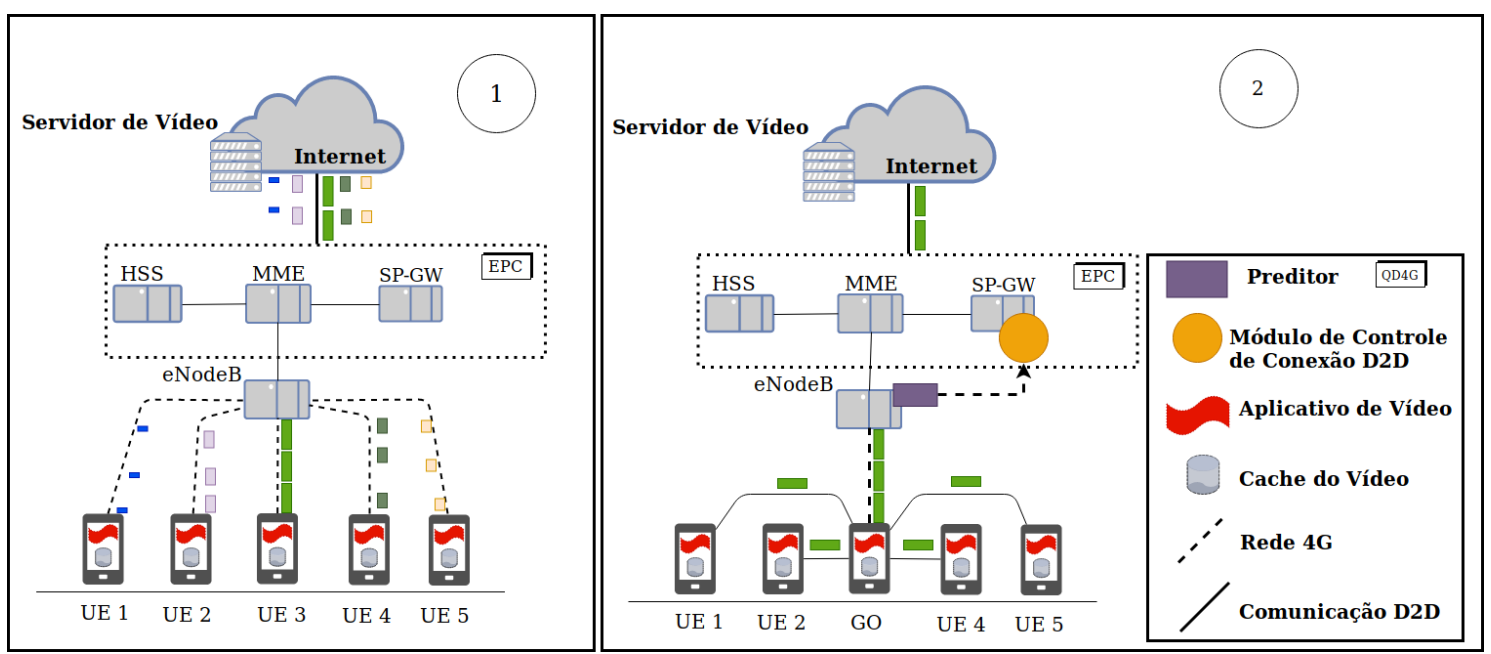

Figura 3. Arquitetura da plataforma QD4G.

causas de degradação de QoE estamos, no nosso ponto de vista, usando um bom indicador da qualidade do vídeo assistido pelo usuário.

A Figura 4 mostra o modelo proposto. O modelo emprega AM supervisionado, para evitar que a aplicação envie dados de retorno ao QD4G. Como a resolução do vídeo é dada por valores discretos, optamos pelo uso de um classificador ao invés de um regressor.

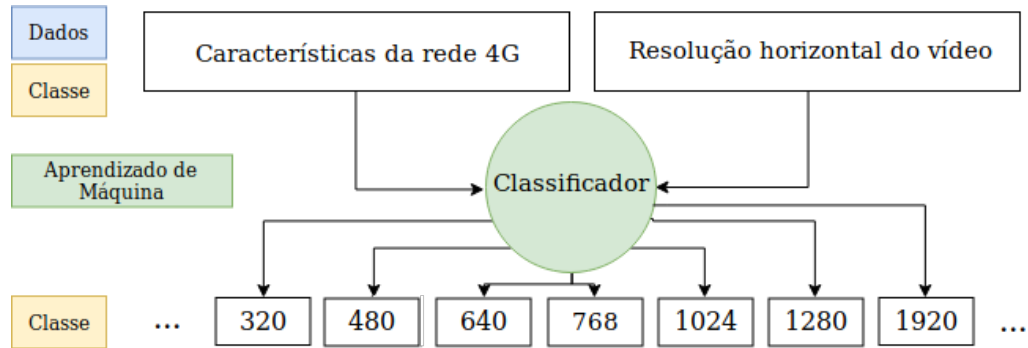

Figura 4. Diagrama do modelo de predição.

A Tabela 2 exibe as características coletadas da rede 4G, as quais são os valores de entrada do modelo de predição. Na Tabela, também é indicado em qual direção da comunicação (downlink - DL ou uplink - UL) as características são coletadas. As entradas são disponibilizadas pelo eNodeB e pelos UEs (resolução horizontal do vídeo), deste modo a solução proposta não demanda alterações no SO ou chipset $4 \mathrm{G}$ dos UEs.

\subsection{Módulo de Controle de Conexão D2D}

O módulo de controle, desenvolvido em Python, é executado no SP-GW em conjunto com o eNodeB, e implementa o algoritmo de decisão.

O Algoritmo 1 exibe o procedimento para criação de um grupo D2D. Cria-se um grupo D2D quando é possível aumentar a resolução do vídeo recebido por algum UE, como estimado pelo preditor. O algoritmo é executado de forma periódica, a intervalos de um segundo, e caso haja ao menos um UE assistindo um dado vídeo com uma resolução inferior à resolução máxima, um grupo é criado. O GO é definido como o UE que recebe o vídeo com a maior resolução (linha 5). Caso haja mais de um UE com a mesma resolução máxima, é escolhido aquele que se conectou primeiro à rede 4G. Para que o algoritmo selecione o GO e comande a troca de interfaces (4G para D2D), o RNTI com maior 
Tabela 2. Características da rede 4G.

\begin{tabular}{|c|c|c|}
\hline Abreviação & Descrição & Direção \\
\hline RNTI & $\begin{array}{c}\text { Radio Network Temporary Identifier - Identificador temporário } \\
\text { para cada dispositivo conectado. }\end{array}$ & DL \\
\hline CQI & $\begin{array}{c}\text { Channel Quality Indicator - Indicador de qualidade do canal. } \\
\text { Esse valor varia de 0 a 15 (quanto maior melhor a qualidade). } \\
\text { Esse dado é informado periodicamente pelo UE ao eNodeB. }\end{array}$ & DL \\
\hline MCS & $\begin{array}{c}\text { Modulation and Coding Scheme - Refere-se ao esquema de mo- } \\
\text { dulação e codificação. Quanto maior o MCS, mais bits podem ser } \\
\text { transmitidos por unidade de tempo. }\end{array}$ & DL \\
\hline BRATE & Taxa de transferência do UE. & DL e UL \\
\hline BLER & $\begin{array}{r}\text { Block Error Rate - É uma relação entre o número de Physical } \\
\text { Resource Blocks (PRBs) perdidos e transmitidos. }\end{array}$ & DL e UL \\
\hline SNR & Relação sinal ruído. & UL \\
\hline PHR & $\begin{array}{c}\text { Power Headroom - Quantidade de potência ainda disponível para } \\
\text { se alcançar a potência máxima frente à potência atual. }\end{array}$ & UL \\
\hline
\end{tabular}

resolução deve se manter estável durante um intervalo de 10 segundos, sendo que esse intervalo foi definido de forma empírica durante os experimentos.

O módulo de gerenciamento de conexão também realiza a volta dos dispositivos que estão em um grupo D2D para a rede 4G. Isso ocorre quando há um grupo D2D formado e a resolução prevista (por meio das medições provenientes do eNodeB) para os UEs configurados como clientes D2D é maior do que a resolução prevista para o UE configurado como GO (linha 9). É importante observar que o módulo de gerenciamento não considera a previsão da resolução diretamente por meio das conexões $\mathrm{D} 2 \mathrm{D}$, sendo que o emprego dessa forma de previsão é considerado aqui como trabalho futuro.

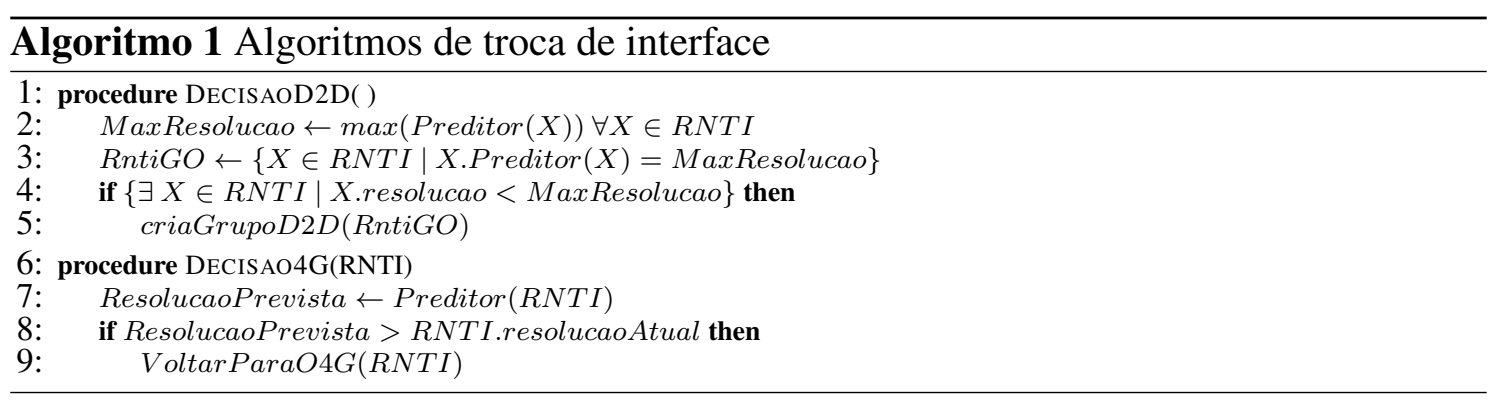

\subsection{Aplicativo de Vídeo}

O aplicativo de vídeo foi alterado para suportar comunicação D2D, pois ele pode requisitar os chunks de dois locais diferentes (servidor de vídeo na Internet ou UE GO do grupo D2D). Além disso, os UEs devem armazenar em cache partes do vídeo, com o objetivo de compartilhar com outros UEs. Assim, foi desenvolvido um aplicativo Android ciente do D2D. Ele é dividido em três módulos, detalhados a seguir.

Módulo de Transmissão e Controle de Vídeo: Reproduz o vídeo sob demanda e salva em cache local este vídeo. Também coleta dados para avaliar o desempenho, como o número de bytes transferidos, a taxa de transferência, o tempo gasto para transferir os bytes (em milissegundos) e a resolução horizontal do vídeo.

Módulo de Conexão D2D: Controla a formação de um grupo D2D, executando as tarefas: $i$ ) Criação do GO (um UE cria um grupo e se declara GO), ii) Busca por 
dispositivos e iii) Conexão com o GO. Um UE com o papel de GO permanece conectado nas redes $4 \mathrm{G}$ e D2D, realiza o download do vídeo pela interface $4 \mathrm{G}$ e transfere via D2D o vídeo para os UEs do grupo D2D.

Cache de chunks: O aplicativo armazena chunks de vídeo recebidos recentemente, para que possam ser repassados para outros UEs, caso o nó seja o GO.

\section{Metodologia de Avaliação e Resultados}

A proposta foi avaliada em um ambiente experimental (testbed), empregando UEs reais e uma rede $4 \mathrm{G}$ montada com software e hardware livres. Há de se considerar que todo testbed possui limitações de escala devido ao seu alto custo. Ainda que possua tais limitações, decidimos rodar os experimentos nesse ambiente com o objetivo de diminuir significativamente a distância entre a nossa proposta e a sua aplicação no mundo real.

Foi construída uma rede $4 \mathrm{G}$ no testbed FUTEBOL $^{6}$ da UFMG. Foram utilizados miniPCs Dell Alienware i7, que possuem rádios definidos por software Ettus USRP de segunda geração conectados via USB (modelos B200 e B210). Foi utilizado o OpenAirInterface $^{7}$ para emular os elementos do núcleo 4G (HSS, MME, SP-GW), e o srsLTE ${ }^{8}$ para emular o eNodeB. A criação do enlace de comunicação $4 \mathrm{G}$ entre o eNodeB e os UEs foi feita por meio do rádio USRP conectado no miniPC do testbed FUTEBOL. Como UEs foram empregados cinco smartphones LG Nexus 5X. O servidor de vídeo foi um $n$ gin $x^{9}$ que é baseado no padrão DASH. Nesse servidor foram disponibilizadas 7 resoluções horizontais diferentes do vídeo "Big Buck Bunny"10 de 320 pixels a 1920 pixels com duração de 10 minutos. Os UEs empregaram o aplicativo Android descrito na seção 4.3.

\subsection{Treinamento do Modelo de Predição}

A primeira fase dos experimentos compreende o treinamento e medição da acurácia do modelo de predição. As instâncias de treino foram obtidas a partir dos UEs assistindo vídeos no testbed FUTEBOL da UFMG, e combinam informações do eNodeB (Tabela 2) e a resolução horizontal do vídeo nos UEs (seção 4.3).

Para a combinação, os relógios foram sincronizados durante a coleta de dados através do protocolo NTP. A coleta foi feita em rodadas decrescentes de cinco UEs até um, recebendo o vídeo apenas através da rede $4 \mathrm{G}$. Cada rodada teve três repetições. O objetivo foi cobrir o maior número de possibilidades comportamentais da rede. As tabelas 3 e 4 apresentam a caracterização dos dados de treino do preditor.

$\mathrm{O}$ aprendizado foi feito no $\mathrm{Weka}^{11}$. Foram realizados experimentos com 5 algoritmos de classificação, como mostra a Figura 5. O custo computacional dos algoritmos avaliados não foi considerado, uma vez que ele se mostrou imperceptível em todos os experimentos.

\footnotetext{
${ }^{6}$ http://www.ict-futebol.org.br - Último acesso em 28/02/2019

${ }^{7}$ http://www.openairinterface.org - Último acesso em 28/02/2019

${ }^{8}$ https://github.com/srsLTE/srsLTE - Último acesso em 28/02/2019

${ }^{9}$ https://www.nginx.com - Último acesso em 28/02/2019

${ }^{10} \mathrm{https}$ ://peach.blender.org/about/ - Último acesso em 28/02/2019

${ }^{11} \mathrm{https}: / /$ www.cs.waikato.ac.nz/ml/weka/ - Último acesso em 28/02/2019
} 
Em seguida, foram avaliados os dois algoritmos com maior porcentagem de acertos na classificação através da ferramenta de software livre scikit-learn ${ }^{12}$, desenvolvida em Python. Para cada experimento, utilizou-se a validação cruzada, onde $80 \%$ dos dados eram usados para treinamento e $20 \%$ para teste e validação. Os resultados indicaram que o algoritmo Random Forest obteve a maior porcentagem de classificação correta nas duas ferramentas. Assim, esse algoritmo foi escolhido para ser avaliado experimentalmente.

A verificação do modelo após o treinamento fornece informações para explicar o seu comportamento e o da rede 4G. A Tabela 5 exibe a relevância de cada característica da rede na classificação e predição da resolução do vídeo. O SNR, BLER e BRATE de downlink são as características que mais contribuem para a precisão do classificador.

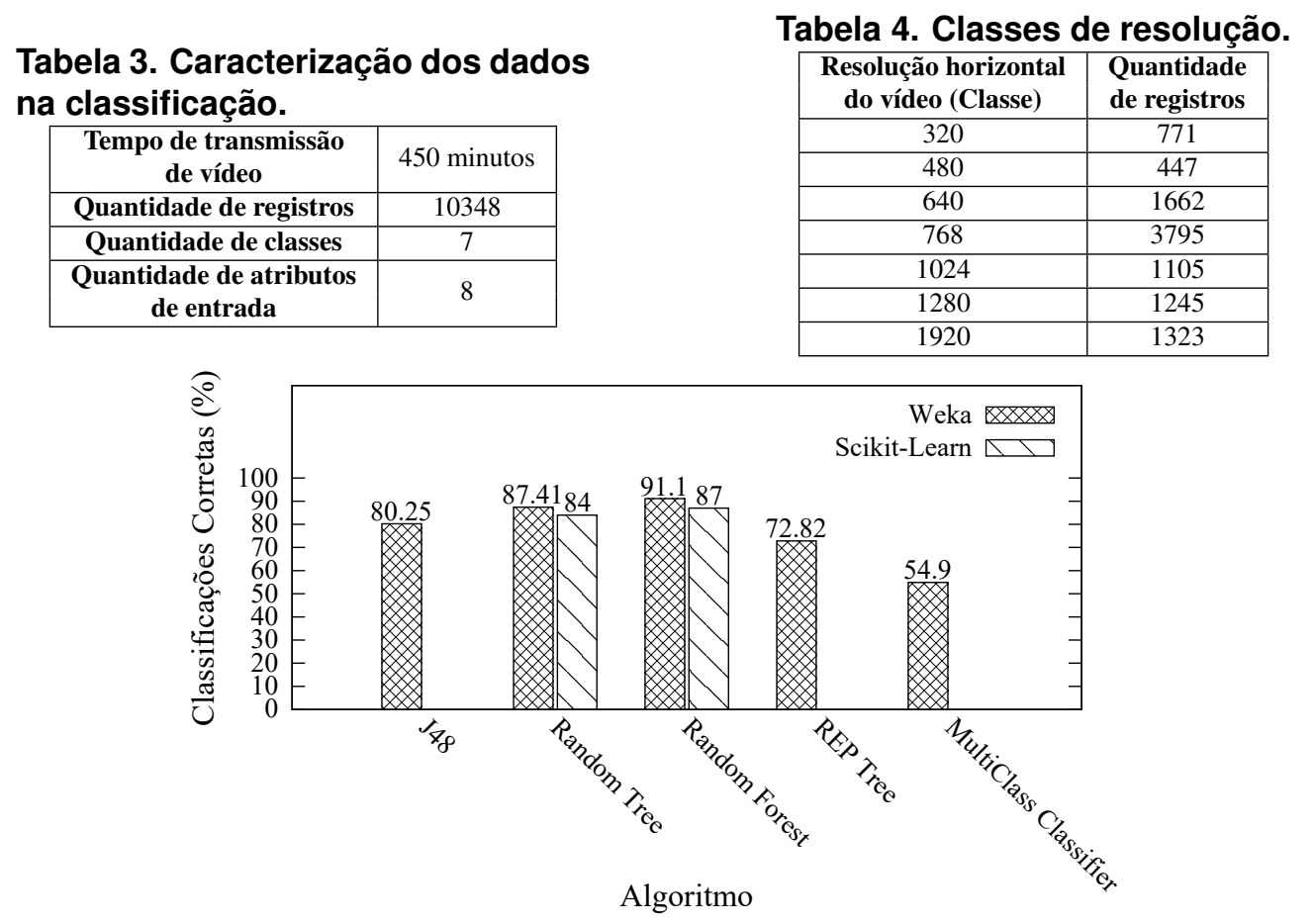

Figura 5. Avaliação dos algoritmos de classificação.

Tabela 5. Importância das características.

\begin{tabular}{|c|c|}
\hline Característica & Importância \\
\hline CQI & 0.07 \\
\hline MCS & 0.09 \\
\hline BRATE (DL) & 0.17 \\
\hline BRATE (UL) & 0.05 \\
\hline BLER (DL) & 0.15 \\
\hline BLER (UL) & 0.10 \\
\hline SNR & 0.20 \\
\hline PHR & 0.08 \\
\hline
\end{tabular}

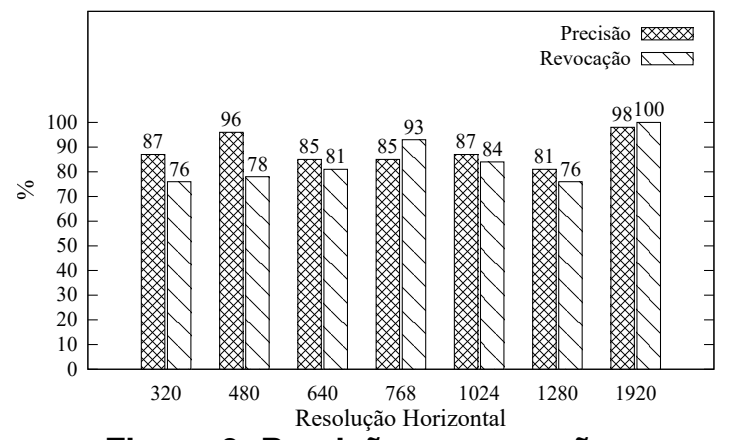

Figura 6. Precisão e revocação.

A Tabela 6 exibe a matriz de confusão do modelo gerado para o Random Forest. Essa Tabela exibe quando uma classe é classificada como pertencente a ela mesma (verdadeiro positivo) ou a outra classe (falso positivo). Já a Figura 6 mostra a porcentagem da precisão e revocação para cada resolução do vídeo, sendo que a precisão é a relação entre o número de exemplos que foram classificados corretamente na classe e o número

\footnotetext{
${ }^{12} \mathrm{https} / / /$ scikit-learn.org/stable/ - Último acesso em 28/02/2019
} 
total de exemplos classificados na classe (verdadeiros positivos + falsos positivos), e a revocação é a relação entre o número de vezes que uma classe foi classificada corretamente e o número de vezes que a classe aparece no conjunto de teste (verdadeiros positivos + falsos negativos). O modelo teve uma revocação acima de $70 \%$ para todas as classes, assim como precisão acima de $80 \%$, com destaque à classe de maior resolução horizontal (1920 pixels), para a qual o modelo mostrou-se extremamente assertivo, garantindo uma precisão de até $98 \%$.

Tabela 6. Matriz de confusão.

\begin{tabular}{|c||c|c|c|c|c|c|c|}
\hline Real Classificação & 320 & 480 & 640 & 768 & 1024 & 1280 & 1920 \\
\hline \hline 320 & 130 & 0 & 9 & 28 & 0 & 4 & 0 \\
\hline 480 & 3 & 68 & 10 & 4 & 5 & 5 & 0 \\
\hline 640 & 2 & 1 & 256 & 54 & 3 & 1 & 0 \\
\hline 768 & 9 & 0 & 12 & 699 & 3 & 21 & 0 \\
\hline 1024 & 3 & 2 & 11 & 6 & 179 & 12 & 0 \\
\hline 1280 & 0 & 0 & 5 & 35 & 16 & 198 & 6 \\
\hline 1920 & 0 & 0 & 0 & 0 & 0 & 1 & 273 \\
\hline
\end{tabular}

\subsection{Avaliação Experimental}

Em seguida, o QD4G é avaliado através de experimentos. Foram realizadas cinco repetições de 10 minutos cada. Foram calculadas as médias da resolução horizontal do vídeo observadas por cada UE, nas cinco repetições realizadas. O intervalo de confiança considerado foi de $95 \%$. O código-fonte do QD4G, utilizado nos experimentos, pode ser obtido através do repositório da plataforma no GitHub ${ }^{13}$.

Como baseline foi considerada uma rede $4 \mathrm{G}$ com todos os UEs baixando o mesmo vídeo, sem empregar a comunicação D2D. É esperado que essa concorrência na rede 4G cause congestionamentos, reduzindo assim o QoE dos UEs.

A Figura 7 mostra a média da resolução horizontal em cada um dos UEs, tanto no baseline quanto na plataforma QD4G. Para todos os UEs, a resolução foi superior em relação ao baseline. Para o UE 3, a resolução horizontal média foi de 625 para 1559, um aumento de cerca de $150 \%$. Isso já era esperado, uma vez que a formação de grupos D2D só será realizada quando for encontrado um UE que possua em seu cache um vídeo com maior resolução que os outros UEs.

Também pode ser observado na Figura 7 que a resolução horizontal média foi a mesma para todos os UEs no QD4G. Isso se deve ao fato de que, durante todos os experimentos, apenas um grupo D2D precisou ser criado, no qual quatro UEs clientes se conectaram a um UE GO para baixar o vídeo, desonerando a rede em $80 \%$. Assim, todos os UEs se mantiveram homogêneos em relação ao QoE, além de reduzir a demanda pelo vídeo na rede $4 \mathrm{G}$, fator que inclusive contribuiu para que o UE selecionado como GO pudesse baixar o vídeo com maior resolução na rede $4 \mathrm{G}$.

Por fim, os intervalos de confiança na Figura 7 exibem maior variação na resolução de alguns UEs do baseline, como por exemplo o UE 4. Essa variação e as diferenças na resolução média observadas entre os UEs do baseline, pode ser explicada pela maior demanda pelo vídeo na rede $4 \mathrm{G}$ em relação ao cenário do QD4G, que possuía apenas um UE baixando o vídeo através da rede 4G. A alta concorrência faz com que o vídeo

\footnotetext{
${ }^{13} \mathrm{https} / / /$ github.com/marcosmagno/QD4G
} 


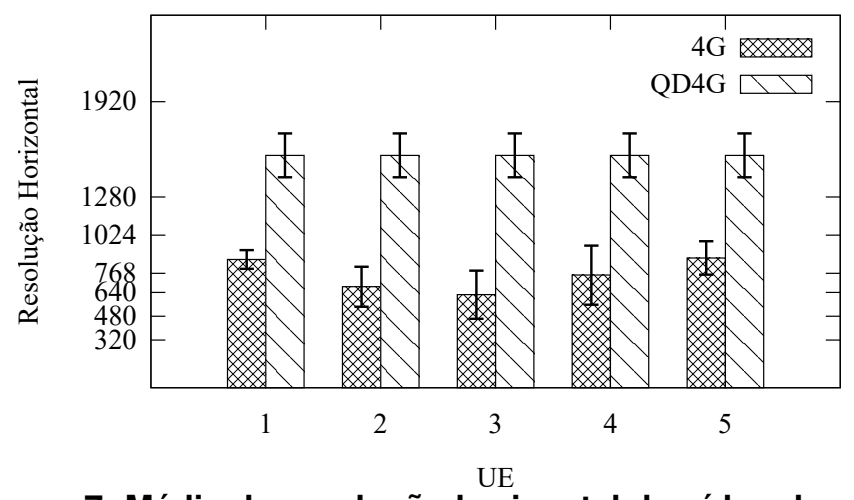

Figura 7. Média da resolução horizontal do vídeo dos UEs.

de cada UE sofra alterações mais frequentes na resolução, o que pode prejudicar o QoE. Esse prejuízo ocorre principalmente quando a variação se encontra em um intervalo de resoluções baixas, como foi o caso do baseline, que oscilou entre as resoluções horizontais de 480 e 768 pixels em sua maioria. No caso do QD4G, ainda que a variação na resolução tenha sido semelhante às que foram observadas no baseline, ela foi constante em todos os UEs, o que mostra que a comunicação D2D tem um comportamento mais previsível. Por fim, é importante observar que a variação do QD4G ocorreu entre resoluções horizontais mais altas, superiores a 1280 pixels, o que reduz o prejuízo ao QoE uma vez que variações nesse intervalo são percebidas com menor facilidade pelos usuários.

A Figura 8 exibe a resolução horizontal do vídeo nos UEs, observada através do QD4G. À esquerda da linha vertical, estão os downloads ainda em andamento na rede 4G, e à direita os downloads feitos via D2D. Durante o download através da rede 4G, a resolução dos UEs muda constantemente, devido à alta concorrência nessa rede. Após um determinado intervalo de tempo, o UE 2 se sobressai com maiores resoluções de vídeo e mantém uma diferença discrepante em relação aos outros. Um dos possíveis fatores para tal ocorrência é o fato de que esse UE possuía melhor conexão com o eNodeB, o que permitiu que resoluções maiores pudessem ser baixadas. Esse fator é observado pelo QD4G, por meio do modelo de predição, que em determinado momento decide criar um grupo D2D, e escolhe o UE 2 como GO, fazendo com que todos os outros UEs baixem o

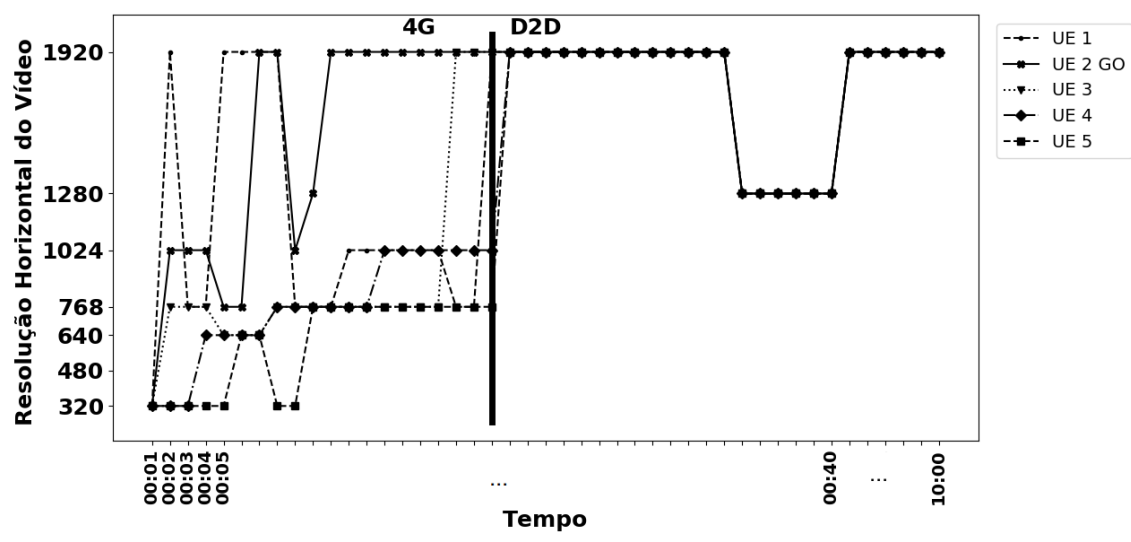

Figura 8. Comportamento da rede. 
vídeo na resolução máxima oferecida, a partir do UE 2, estabilizando todos nesse patamar. Desta forma, a resolução horizontal do vídeo para os clientes segue o padrão do GO. Durante a transmissão via D2D, há variações na resolução do vídeo entregue ao GO, contudo, essa resolução se manteve superior em relação ao intervalo em que todos os UEs estão na rede $4 \mathrm{G}$, como mostrado na Figura 8. Para fins de simplificação, essa variação foi ocultada a partir do tempo 00:40.

\section{Conclusão e Trabalhos Futuros}

Este trabalho apresentou o QD4G, uma plataforma que emprega grupos D2D para aumentar o QoE de aplicações de vídeo em redes 4G. A decisão para criar um grupo D2D é baseada em aprendizado de máquina. Os resultados mostraram uma precisão de até $87 \%$, além de um aumento de $150 \%$ na resolução média do vídeo. Devido a limitações de hardware presentes no ambiente de experimentação, não foi possível avaliar o desempenho da plataforma considerando cenários com maior quantidade de UEs.

Como trabalhos futuros, pretendemos avaliar a qualidade do canal D2D entre o GO e os UEs conectados a ele. Com isso, evitamos casos em que um UE tenha uma conexão D2D de baixa vazão com o GO. Também pretendemos avaliar o aumento médio da vazão dos UEs, em casos que os mesmos estão conectados em grupos D2D, comparando essa vazão frente à observada na rede 4G. Por fim, serão feitas avaliações focadas no consumo de energia, uma vez que a comunicação D2D é mais simples e gasta menos energia em relação à comunicação 4G [Pyattaev et al. 2013]. Até onde sabemos, não há trabalhos relacionados onde o operador é capaz de obter o nível da bateria dos UEs. Portanto, uma regra simples seria desconsiderar UEs com pouca bateria na escolha do GO.

\section{Agradecimentos}

Este trabalho foi desenvolvido por meio de recursos provenientes das agências de fomento CAPES, CNPq, Fapemig, e o projeto FUTEBOL. O projeto FUTEBOL recebeu recursos do programa Horizon 2020 da União Europeia, via contrato número 688941 (FUTEBOL), bem como recursos do Ministério de Ciência Tecnologia, Inovação e Comunicação (MCTIC) via RNP e CTIC.

\section{Referências}

[Asadi et al. 2014] Asadi, A., Wang, Q., and Mancuso, V. (2014). A Survey on Device-toDevice Communication in Cellular Networks. IEEE Communications Surveys Tutorials, 16(4):1801-1819.

[Bradai et al. 2015] Bradai, A., Singh, K., Ahmed, T., and Rasheed, T. (2015). Cellular Software Defined Networking: a Framework. Communications Magazine, IEEE, 53(6):3643.

[Camps-Mur et al. 2013] Camps-Mur, D., Garcia-Saavedra, A., and Serrano, P. (2013). Device-to-Device Communications with Wi-Fi Direct: Overview and Experimentation. IEEE Wireless Communications, 20(3):96-104.

[Chai et al. 2016] Chai, X., Liu, T., Xing, C., Xiao, H., and Zhang, Z. (2016). Throughput improvement in cellular networks via full-duplex based device-to-device communications. IEEE Access, 4:7645-7657. 
[Doppler et al. 2009] Doppler, K., Rinne, M., Wijting, C., Ribeiro, C. B., and Hugl, K. (2009). Device-to-Device Communication as an Underlay to LTE-Advanced Networks. IEEE Communications Magazine, 47(12).

[Essaili et al. 2015] Essaili, A. E., Schroeder, D., Steinbach, E., Staehle, D., and Shehada, M. (2015). QoE-Based Traffic and Resource Management for Adaptive HTTP Video Delivery in LTE. IEEE Transactions on Circuits and Systems for Video Technology, 25(6):988-1001.

[Haldar et al. 2013] Haldar, K. L., Li, H., and Agrawal, D. P. (2013). A Cluster-Aware Soft Frequency Reuse Scheme for Inter-Cell Interference Mitigation in LTE Based Femtocell Networks. In World of Wireless, Mobile and Multimedia Networks (WoWMoM), 2013 IEEE 14th International Symposium and Workshops on a, pages 1-6. IEEE.

[Kua et al. 2017] Kua, J., Armitage, G., and Branch, P. (2017). A survey of rate adaptation techniques for dynamic adaptive streaming over http. IEEE Communications Surveys Tutorials, 19(3):1842-1866.

[Liu et al. 2015] Liu, J., Kato, N., Ma, J., and Kadowaki, N. (2015). Device-to-Device Communication in LTE-Advanced Networks: A Survey. IEEE Communications Surveys Tutorials, 17(4):1923-1940.

[Mi et al. 2014] Mi, X., Tian, Z., Xu, X., Zhao, M., and Wang, J. (2014). NO stack: A SDNBased Framework for Future Cellular Networks. In Wireless Personal Multimedia Communications (WPMC), 2014 International Symposium on, pages 497-502.

[Mota et al. 2013] Mota, V. F. S., Macedo, D. F., Ghamri-Doudane, Y., and Nogueira, J. M. S. (2013). On the Feasibility of WiFi Offloading in Urban Areas: The Paris Case Study. In IFIP Wireless Days conference.

[Pyattaev et al. 2013] Pyattaev, A., Johnsson, K., Andreev, S., and Koucheryavy, Y. (2013). 3GPP LTE traffic offloading onto WiFi Direct. In 2013 IEEE Wireless Communications and Networking Conference Workshops (WCNCW), pages 135-140.

[Sesia et al. 2009] Sesia, S., Toufik, I., and Baker, M. (2009). LTE, The UMTS Long Term Evolution: From Theory to Practice. Wiley Publishing.

[Stockhammer 2011] Stockhammer, T. (2011). Dynamic Adaptive Streaming over HTTP-: Standards and Design Principles. In Proceedings of the Second Annual ACM Conference on Multimedia Systems, pages 133-144. ACM.

[Thrimurthulu and Sarma 2017] Thrimurthulu, V. and Sarma, N. M. (2017). Device-todevice Communications in Long Term Evaluation-Advanced Network. In Intelligent Computing and Control Systems (ICICCS), 2017 International Conference on, pages 818-823. IEEE.

[Ullah and Hong 2016] Ullah, S. and Hong, C. S. (2016). Outband D2D Communication for Layered Video Delivery in ICN Enabled Cellular Network. Proceedings of the Korean Information Science Society, pages 1370-1372.

[Xu et al. 2018] Xu, Y., Jiang, S., and Wu, J. (2018). Towards Energy Efficient Device-toDevice Content Dissemination in Cellular Networks. IEEE Access, 6:25816-25828.

[Zhou et al. 2018] Zhou, H., Wang, H., Li, X., and Leung, V. C. M. (2018). A Survey on Mobile Data Offloading Technologies. IEEE Access, 6:5101-5111. 